\title{
A Lossless IMAGE COMPRESSION USING TrADITIONAL AND LIFTING BASED WAVELETS
}

\author{
Dr.B Eswara Reddy ${ }^{1}$ and K Venkata Narayana ${ }^{2}$ \\ ${ }^{1}$ Dept of CSE, JNT University Anantapur, Anantapur, India \\ eswarcsejntu@gmail.com \\ ${ }^{2}$ Dept of CSE, SKU College of Engg \& Tech, SKUniversity, Anantapur, India \\ venkat2008.sku@gmail.com
}

\begin{abstract}
In this paper an attempt has been made to analyse different wavelet techniques for image compression. Both hand-designed and lifting based wavelets are considered. Hand designed wavelets considered in this work are Haar wavelet, Daubechie wavelet, Biorthognal wavelet, Demeyer wavelet, Coiflet wavelet and Symlet wavelet. Lifting based wavelet transforms considered are 5/3 and 9/7. Wide range of images, including both color and gray scale images were considered. These wavelet transforms are used to compress the test images competitively by using Set Partitioning In Hierarchical Trees (SPIHT) algorithm and by incorporating lifting concepts. Set Partitioning In Hierarchical Trees is a new advanced algorithm based on wavelet transform which is gaining attention due to many potential commercial applications in the area of image compression. These algorithms resulted in practical advantages, such as, superior low bit rate performance, bit-level compression, progressive transmission by pixel, accuracy and resolution .The SPIHT coder is also a highly refined version of the EZW algorithm and is a powerful image compression algorithm, that produces an embedded bit stream form, in which the best reconstructed images shows a significant perceptual improvement as well as an increased PSNR.
\end{abstract}

\section{KEYWORDS}

Wavelet, Image Compression, SPIHT, Lifting, Hand-designed

\section{INTRODUCTION}

Image compression is one of the most important and successful applications of the wavelet transform. Mature wavelet based image coders like the JPEG2000 standard [1] are available, gaining popularity, and easily outperform traditional coders based on the discrete cosine transform (DCT) like JPEG [2]. Unlike in DCT based image compression, however, the performance of a wavelet based image coder depends to a large degree on the choice of the wavelet. This problem is usually handled by using standard wavelets that are not specially adapted to a given image, but that are known to perform well on photographic images. However, many common classes of images do not have the same statistical properties as photographic images, such as fingerprints, medical images, scanned documents and satellite images. The standard wavelets used in image coders often do not match such images resulting in decreased compression or image quality. Moreover non-photographic images are often stored in large databases of similar images, making it worthwhile to find a specially adapted wavelet for them. A Novel data-pruning based compression scheme to reduce bit rate while still keeping a high quality reconstructed frame [3].

Memory and bandwidth are the prime constraints in image storage and transmission applications. One of the major challenges in enabling mobile multimedia data services will be the need to

DOI : $10.5121 /$ sipij.2012.3216 
Signal \& Image Processing : An International Journal (SIPIJ) Vol.3, No.2, April 2012

process and wirelessly transmit a very large volume of data. While significant improvements in achievable bandwidth are expected with future wireless access technologies, improvements in battery technology will lag the rapidly growing energy requirements of future wireless data services. One approach to mitigate this problem is to reduce the volume of multimedia data transmitted over the wireless channel via data compression techniques. This has motivated active research on multimedia data compression techniques such as JPEG [4, 5], JPEG $2000[6,7]$ and MPEG [8]. These approaches concentrate on achieving higher compression ratio without sacrificing the quality of the image. However these efforts ignore the energy consumption during compression and RF transmission. Since images will constitute a large part of future wireless data, the thesis aim on developing energy efficient and adaptive image compression and communication techniques. Based on wavelet image compression, energy efficient multi-wavelet image transform is a technique developed to eliminate computation of certain high-pass coefficients of an image.

\section{RELATED WORK}

Any type of data transmission or storage without compression is impractical for the following reasons.

- The data handled by different digital environments is increasing at a rate more than twice a year.

- The storing of digital data without compression would be a tragedy.

- Similar to the case of storage, the transmission is the other concern. With the insight into the World Wide Web (WWW), where more than 7500 Tera Bytes of data is being downloading and/or uploading, the transmission is the major concern in the modern world.

Hence it was made mandatory to have compression in one form or other. To incorporate compression or even to modify any image in any sense, frequency domain is assumed to be more convenient. To transform an image from spatial domain to frequency domain, the basic technique that was in use is Discrete Cosine Transform (DCT). DCT is very simple technique with low complexity. But it has the following pitfall.

- Only spatial correlation of the pixels inside the single 2D block is considered, and the correlation from the pixels of the neighboring blocks is neglected. It results blocking artifacts. If it so, the human interpreter may not identify image properly.

After the mention of Wavelet by Haar in his Ph.D thesis in 1909, Discrete Wavelet Transform (DWT) was used in most of the image compression applications as it overcomes the disadvantages of DCT. But it too has the following limitations.

- Computational Complexity

- Memory Complexity

- Low image quality

- Floor operating losses

- Poor compression ratio

- Low bit degradation

Wavelet transforms have received significant attention in many fields, such as mathematics, digital signal and image processing, because of their ability to represent and analyze data. This work is intended to summarize important developments and recent progress in the areas where novel wavelet processing concepts are incorporated into optics research [9] and we present the 
Signal \& Image Processing : An International Journal (SIPIJ) Vol.3, No.2, April 2012

construction of adaptive wavelets by means of an extension of the lifting scheme [10]. The basic idea is to choose the update filters according to some decision criterion which depends on the local characteristics of the input signal. We show that these adaptive schemes yield lower entropies than schemes with fixed update filters, a property that is highly relevant in the context of compression. This work also describes a low cost board to support a video compression, restoration and filters system in real time processing [11]. This wave Net board has been optimized for wavelet-based image and video compression and enhancement techniques. The algorithm for the Non-Standard Decomposition and the Standard Decomposition are both of outstandingly low computational complexity with the Non-Standard being slightly quicker.

The results show that the Non Standard Decomposition in conjunction with the Haar Wavelet is an excellent choice as the algorithmic base for a software-only codec [12]. The wavelet transform, defined by Yves Meyer and J. Lemarie offers good localization in both, space and frequency domains and can be implemented by fast algorithms. Since the discrete wavelet transform (DWT) was presented by Mallat, many researchers on signal analysis and image compression have derived fruitful results due to its well time-frequency decomposition. Recently, a new wavelet construction called lifting scheme, has been developed by Wim Sweldens and Ingrid Daubechies [13]. It has other applications, such as the possibility of defining a wavelet-like transform that maps integers to integers [14]. This method has gained increasing interest in scientific community, due to its reduced computational complexity by first factoring a classical wavelet filter into lifting steps.

A number of adaptive wavelet transforms based on the lifting scheme have been proposed in the literature. Taubman [15] developed adaptive wavelet transforms to modify the prediction step by using the properties of the image. Since this predictor takes into account the fact that the discontinuities in images tend to occur along continuous curves, such adaptation of the predictor makes the transform non-linear [16] and [17]. Exploiting local orientation information at edge boundaries, Calypoole et al. [18] have proposed a prediction operator based on the local properties of the image. In [19] they described an adaptive polyphase structure based on the reduction of the variance. In [20] they first calculate the optimal predictors, by minimizing the prediction error variance, and then they apply these optimal predictor filters with adaptive update filters.

This work is based on applying the update adaptive wavelet filter presented in [21] to lossy image compression. The approach taken in this paper differs from other adaptive schemes found in the literature in the sense that no book keeping is required in order to have perfect reconstruction. The choice of the update-lifting filter is triggered by a binary threshold criterion based on a generalized gradient that is chosen in such a way that it only smooths homogeneous regions [22]. In this paper, we present 5/3 and 9/7 lifting based wavelet transforms for improved image compression.

\section{DESIGN METRICS}

Digital image compression techniques are examined with various metrics. Among those the most important one is Peak Signal to Noise Ratio (PSNR) which will express the quality. There exists another property which expresses the quality, that is, Mean Square Error (MSE). PSNR is inversely proportional to MSE. The other important metric is Compression Ratio, which express the amount of compression embedded in the technique. In theory, it was observed that PSNR and Compression ratios are inversely related. The other metrics are Encoding Time, Decoding Time and Transforming Time. 
Mean Square Error: Among the quantitative measures, a class of criteria used often is called the mean square criteria. It refers to some sort of average or sum (or integral) of squares of the error between two images. MSE for monochrome image

MSE for color image

$$
\frac{1}{N^{2}} \sum_{i}^{N} \sum_{j}^{N}(X(i, j)-Y(i, j))^{2}
$$

$$
\frac{1}{N^{2}} \sum_{i}^{N} \sum_{j}^{N}\left[\left(r(i, j)-r^{*}(i, j)\right)^{2}+\left(g(i, j)-g^{*}(i, j)\right)^{2}+\left(b(i, j)-b^{*}(i, j)\right)^{2}\right]
$$

where $r(i, j), g(i, j)$ and $b(i, j)$ represents a color pixel in location(i,j) of the original image, $r^{*}(i, j)$, $g^{*}(i, j)$ and $b^{*}(i, j)$ represents color pixel of the reconstructed image and $N X N$ denotes the size of the pixels of these color images.

Signal to Noise Ratio: In many applications the Mean Square Error is expressed in terms of a Signal to Noise Ratio (SNR) which is defined in decibels (dB) as

$$
\mathrm{SNR}=10 \log \frac{\sigma^{2}}{\sigma_{e}^{2}}
$$

Where $\sigma^{2}$ is the variance of the desired image and $\sigma_{e}^{2}$ is average variance.

Peak Signal to Noise Ratio: Peak Signal to Noise Ratio is defined as the ratio between signal variance and reconstruction error variance. Mean Square Error, Peak Signal to Noise Ratio and Compression Ratios are calculated from the following expressions.

$$
\mathrm{PSNR}=10 \log _{10} \frac{255^{2}}{M S E}
$$

Compression Ratio: Compression ratio is defined as the ratio between the original image size and compressed image size.

$$
\text { Compression Ratio }=\frac{\text { Original }- \text { image }- \text { size }}{\text { Compressed }- \text { image }- \text { size }}
$$

Encoding Time, Decoding Time and Transforming Time: Any compression system uses one of the encoding techniques to encode the input information. The encoding operation is very crucial for the success of the compression system. It involves the representation of the input information in a form suitable for storage and transmission. The time required to perform this operation is referred to as encoding time. The reverse process to encoding is decoding and the corresponding time required to decode an encoded data is decoding time. In general, the information to be compressed will be represented in time or spatial domain. To compress the data, it was observed that it is convenient to represent the data in frequency domain. Hence the information in time domain needs to be converted into frequency domain. For that, one of the transforming techniques will be used. Again it involves some consumption of time. This time is referred to as transforming time. These times are measured in seconds.

\section{Traditional WaVELets}

Haar wavelet: The Haar sequence was proposed in 1909 by Alfred Haar (79). Haar used these functions to give an example of a countable orthonormal system for the space of square- 
Signal \& Image Processing : An International Journal (SIPIJ) Vol.3, No.2, April 2012

integrable functions on the real line. The study of wavelets, and even the term "wavelet", did not come until much later. As a special case of the Daubechies wavelet, it is also known as D2. The Haar wavelet is also the simplest possible wavelet. The technical disadvantage of the Haar wavelet is that it is not continuous, and therefore not differentiable. This property can, however, be an advantage for the analysis of signals with sudden transitions, such as monitoring of tool failure in machines.

Daubechie wavelet: In general the Daubechies wavelets are chosen to have the highest number A of vanishing moments, (this does not imply the best smoothness) for given support width $\mathrm{N}=2 \mathrm{~A}$, and among the $2^{\mathrm{A}-1}$ possible solutions the one is chosen whose scaling filter has external phase. The wavelet transform is also easy to put into practice using the fast wavelet transform. Daubechies wavelets are widely used in solving a broad range of problems, e.g. self-similarity properties of a signal or fractal problems, signal discontinuities, etc.

Biorthogonal Wavelet: Biorthogonal wavelets extend the families of orthogonal wavelets. It is a well-known fact in the filter theory community that symmetry and perfect reconstruction are incompatible (except for the Haar wavelet) when the same FIR filters are used for decomposition and for reconstruction process. To circumvent this difficulty two wavelets are introduced instead of one.

Coiflet wavelet: Coiflets are discrete wavelets designed by Ingrid Daubechies, at the request of Ronald Coifman, to have scaling functions with vanishing moments. The wavelet is near symmetric, their wavelet functions have $N / 3$ vanishing moments and scaling functions $N / 3-1$, and has been used in many applications using Calderon-Zygmund Operators. Both the scaling function (low-pass filter) and the wavelet function (High-Pass Filter) must be normalised by a factor $\frac{1}{\sqrt{2}}$. Below are the coefficients for the scaling functions for C6-30. The wavelet coefficients are derived by reversing the order of the scaling function coefficients and then reversing the sign of every second one (i.e. C6 wavelet $=\{-0.022140543057,0.102859456942$, $0.544281086116,-1.205718913884,0.477859456942,0.102859456942\})$. Mathematically, this looks like $B_{k}=(-1)^{k} C_{N-1-k}$ where $k$ is the coefficient index, $B$ is a wavelet coefficient and $C$ a scaling function coefficient. $N$ is the wavelet index, i.e. 6 for C6.

Symlet wavelet: Symlets constitute a family of almost symmetric wavelets proposed by Daubechies by modifying the construction of the $d b N$. Apart from the symmetry, the other properties of the two families are similar. Symlets of orders 2 to 8 (syml is simply the Haar wavelet). The idea of construction consists of re-using the $m_{0}$ function introduced for $d b N$, considering $\left|\mathrm{m}_{0}(\mathrm{w})\right|^{2}$ as a function $W$ of the variable $z=e^{i w}$. We can factorize $W$ in various manners in the form of $W(z)=U(z) U\left(\mathrm{z}^{-1}\right)$, since the roots of $W$ with module different from 1 go in pairs: if $z_{l}$ is a root then $z_{l}^{-1}$ is also a root. By constructing $U$ so that its roots are all of module $<1$ we construct the Daubechies wavelets $d b N$. The filter $U$ has a minimal phase. Another option, attained by optimizing factorization so that the filter $U$ has an almost linear phase, produces much more symmetric filters: the symlets.

\section{5/3 AND 9/7 LIFTING BASED WAVELET TRANFORMS}

Every finite filter wavelet can be factored into lifting steps, and the lifting strategy is a highly non-unique process. The basic principle of the lifting scheme is to break up the polyphase matrices for the wavelet filters into a sequence of upper and lower triangular matrices and convert the filter implementation into banded matrix multiplication. 
The conventional lifting algorithm for $5 / 3$ and $9 / 7$ wavelet filters: The $5 / 3$ wavelet transform is adopted in JPEG2000 standard to implement lossless compression of image, which can be obtained by one stage of lifting. The conventional lifting factorization for the forward transform of $5 / 3$ filters is given.

$$
\bar{p}_{1}(z)=\left[\begin{array}{cc}
1 & \alpha\left(1+z^{-1}\right) \\
0 & 1
\end{array}\right]\left[\begin{array}{cc}
1 & 0 \\
\beta(1+z) 0 & 1
\end{array}\right]
$$

Where $\alpha=-1 / 2, \beta=1 / 4$. The implementation of $5 / 3$ wavelet transform can also be represented by using mathematical notations as follows:

$$
\begin{aligned}
& \mathrm{H}(\mathrm{n})=\mathrm{x}(2 \mathrm{n}+1)+\alpha(\mathrm{x}(2 \mathrm{n})+\mathrm{x}(2 \mathrm{n}+2)) \\
& \mathrm{L}(\mathrm{n})=\mathrm{x}(2 \mathrm{n})+\beta(\mathrm{H}(\mathrm{n})+\mathrm{H}(\mathrm{n}-1))
\end{aligned}
$$

where $\mathrm{H}$ and $\mathrm{L}$ represent the high and low frequency components of input signal respectively. $\mathrm{CDF}(9-7)$ wavelet transform is commonly regarded to have good performance in image compression and released as a default for wavelet transform in JPEG2000.Its analysis filter $\bar{h}(n)$ has 9 coefficients, while synthesis filter $\mathrm{h}(\mathrm{n})$ has 7 coefficients, both high-pass filters $\bar{g}(n)$ and $g(n)$ hold 4 order vanishing moments. The analysis filter can be factorized as follow:

$$
\bar{p}_{2}(z)=\left[\begin{array}{cc}
1 & \alpha\left(1+z^{-1}\right) \\
0 & 1
\end{array}\right]\left[\begin{array}{cc}
1 & 0 \\
\beta(1+z) & 1
\end{array}\right]\left[\begin{array}{cc}
1 & \gamma\left(1+z^{-1}\right. \\
0 & 1
\end{array}\right]\left[\begin{array}{cc}
1 & 0 \\
\delta(1+z) & 1
\end{array}\right]\left[\begin{array}{cc}
\varsigma & 0 \\
0 & 1 / \varsigma
\end{array}\right]
$$

where $\alpha, \beta, \gamma, \delta$ and $\varsigma$ are constant values.

Hence, it is indicated that $\operatorname{CDF}(9 / 7)$ wavelet transform can be obtained by using two lifting steps with an additional scale multiplication, and similarly can leads to the following implementation by using mathematical notations:

$$
\begin{gathered}
\mathrm{L}^{(0)}(\mathrm{n})=\mathrm{x}(2 \mathrm{n}), \mathrm{H}^{(0)}(\mathrm{n})=\mathrm{x}(2 \mathrm{n}+1) \\
\mathrm{H}^{(1)}(\mathrm{n})=\mathrm{H}^{(0)}(\mathrm{n})+\alpha\left(\mathrm{L}^{(0)}(\mathrm{n})+\mathrm{L}^{(0)}(\mathrm{n}+1)\right) \\
\mathrm{L}^{(1)}(\mathrm{n})=\mathrm{L}^{(0)}(\mathrm{n})+\beta\left(\mathrm{H}^{(1)}(\mathrm{n})+\mathrm{H}^{(1)}(\mathrm{n}-1)\right) \\
\mathrm{H}^{(2)}(\mathrm{n})=\mathrm{H}^{(1)}(\mathrm{n})+\gamma\left(\mathrm{L}^{(1)}(\mathrm{n})+\mathrm{L}^{(1)}(\mathrm{n}+1)\right) \\
\mathrm{L}^{(2)}(\mathrm{n})=\mathrm{L}^{(1)}(\mathrm{n})+\delta\left(\mathrm{H}^{(2)}(\mathrm{n})+\mathrm{H}^{(2)}(\mathrm{n}-1)\right) \\
\mathrm{H}(\mathrm{n})=\mathrm{H}^{(2)}(\mathrm{n}) / \varsigma, \mathrm{L}(\mathrm{n})=\varsigma \mathrm{L}^{(2)}(\mathrm{n})
\end{gathered}
$$

Where $\mathrm{H}$ and $\mathrm{L}$ represent also the high and low frequency component of input signal respectively. The Discrete Wavelet Transform (DWT) has gained wide popularity due to its excellent decorrelation property, as a consequence many modern image and video compression systems embody the DWT as transform stage.

It is widely recognized that the 9/7 filters are among the best filters for wavelet based image compression. In fact the JPEG2000 image coding standard employs the 9/7 filters as the default wavelet filters for lossy compression, fostering many research efforts in the development of fast and efficient hardware codes. The performance of a hardware implementation of the 9/7 filter bank depends on the accuracy with which filter coefficients are represented. However high precision representation increases hardware resource and processing time. To reduce the complexity of the 9/7 filters the lifting scheme can adopted. Unfortunately the lifting scheme increases hardware timing accumulation due to its serial nature, so that for certain applications it 
cannot be employed. The flipping structure is an attractive alternative to the standard lifting scheme DWT, since it reduces timing accumulation, however it still requires multiplication.

Complexity reduction can be achieved resorting to a filter bank implementation, in particularly very good results can be obtained with the cascaded method proposed in [23]. The basic idea described in [23] is to minimize the number of bit required to represent the $9 / 7$ coefficients. Since this operation would move filters zeros from their original position, the authors modify some terms to account for zeros compensation. Currently the compatibility of low complexity $9 / 7$ filters implementation with floating point ones has not been stressed yet.

\section{Simulation Results:}

In this section, different types of wavelets are considered for image compression. Here the major concentration is to verify the comparison between Hand designed wavelets and Lifting based wavelets. Hand designed wavelets considered in this work are Haar wavelet, Daubechie wavelet, Biorthognal wavelet, Demeyer wavelet, Coiflet wavelet and Symlet wavelet. Lifting based wavelet transforms considered are 5/3 and 9/7. Wide range of images, including both colour and gray scale images were considered. The algorithms are implemented in MATLAB. The Cameraman images experimental results are plotted in figure1.1 to 1.8. A Number of test images are considered and the results on cameraman image were presented in the table 1 . The performance of Hand designed and lifting based wavelet transforms on Cameraman images was analyzed and plotted in figure 2 .

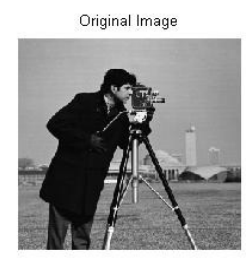

Fig 1.1 Haar Wavelet

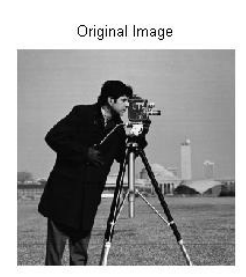

Fig 1.3 Biorthogonal Wavelet
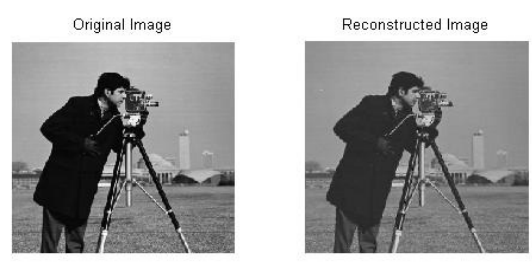

Fig 1.5 Symlet wavelet

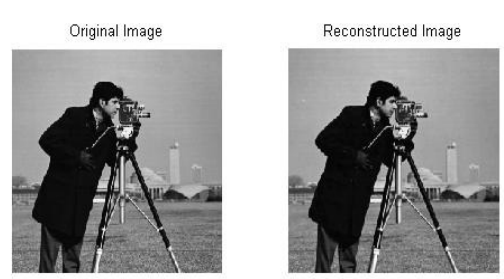

Fig 1.2 Daubechie Wavelet

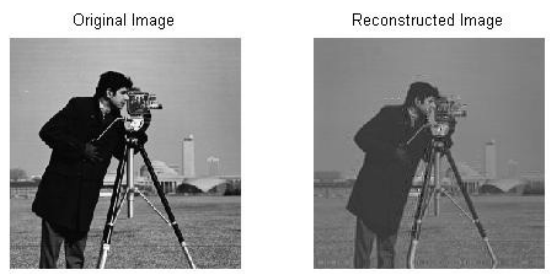

Fig 1.4 Coiflet Wavelet
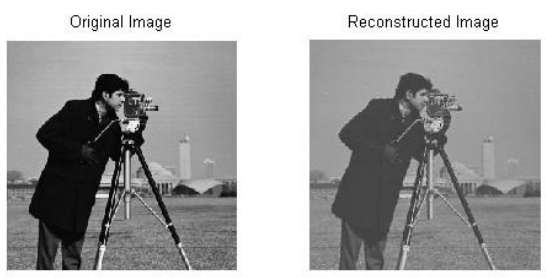

Fig 1.6 Dymer Wavelet 
Signal \& Image Processing : An International Journal (SIPIJ) Vol.3, No.2, April 2012
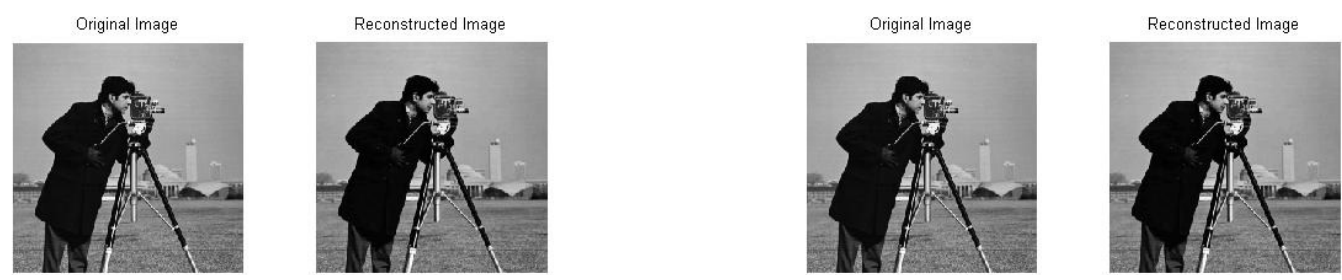

Fig 1.7 5/3 Lifting Based Wavelet

Fig 1.8 9/7 Lifting Based Wavelet

\begin{tabular}{|c|c|c|c|c|c|c|c|c|c|}
\hline \multirow[b]{2}{*}{$\begin{array}{l}\text { INPUT } \\
\text { IMAGE }\end{array}$} & \multicolumn{7}{|c|}{ HAND DESIGNED WAVELETS } & \multicolumn{2}{|c|}{$\begin{array}{l}\text { LIFTING BASED } \\
\text { WAVELET } \\
\text { TRANSFORMS } \\
\end{array}$} \\
\hline & \begin{tabular}{|c||} 
PERFORMA \\
NCE \\
CRITERION
\end{tabular} & HAAR & $\begin{array}{c}\text { DAUBEC } \\
\text { HIE }\end{array}$ & $\begin{array}{l}\text { BIORTHOG } \\
\text { ONAL }\end{array}$ & $\begin{array}{c}\text { DEMEYE } \\
\mathbf{R}\end{array}$ & COIFLET & SYMLET & $\begin{array}{c}5 / 3 \\
\text { TRANSF } \\
\text { ORM }\end{array}$ & $\begin{array}{c}9 / 7 \\
\text { TRANSF } \\
\text { ORM }\end{array}$ \\
\hline \multirow{8}{*}{$\begin{array}{l}\text { CAMERA } \\
\text { MAN } \\
\text { (Gray) }\end{array}$} & $\begin{array}{c}\text { ENC_TIME } \\
\text { (SEC) }\end{array}$ & 6.0226 & 6.6047 & 6.3633 & 7.2604 & 8.1205 & 7.0007 & 6.9664 & 6.6507 \\
\hline & $\begin{array}{l}\text { DEC_TIME } \\
\text { (SEC) }\end{array}$ & 0.8724 & 0.94074 & 0.90272 & 1.1382 & 1.1428 & 1.0361 & 1.1418 & 1.4065 \\
\hline & \begin{tabular}{|c} 
TRANS_TIM \\
E \\
(SEC)
\end{tabular} & 0.061623 & 0.1072 & 0.071691 & 0.27447 & 0.19392 & 0.10731 & 0.16648 & 0.20735 \\
\hline & $\begin{array}{c}\text { ORG_SIZE } \\
\text { (BITS) }\end{array}$ & 524288 & 524288 & 524288 & 524288 & 524288 & 524288 & 1048576 & 1048576 \\
\hline & $\begin{array}{c}\text { COMP_SIZE } \\
\text { (BITS) }\end{array}$ & 212994 & 238939.5 & 233163.5 & 437846.5 & 277302.5 & 250446.5 & 131427.5 & 106116 \\
\hline & $\underset{10}{\text { COMP_RAT }}$ & 2.4615 & 2.1942 & 2.2486 & 1.1974 & 1.8907 & 2.0934 & 7.9784 & 9.8814 \\
\hline & MSE(dB) & 5.91496 & 6.625 & 9.4120 & 7.1211 & 3.20903 & 6.90566 & 7.32437 & 12.3477 \\
\hline & PSNR(dB) & 29.5887 & 30.0811 & 31.606 & 30.3947 & 46.9329 & 30.2612 & 39.51712 & 37.2489 \\
\hline
\end{tabular}

TABLE 1: Performance comparison between Hand designed and Lifting based wavelet transforms on 'Cameraman' (Gray) image.

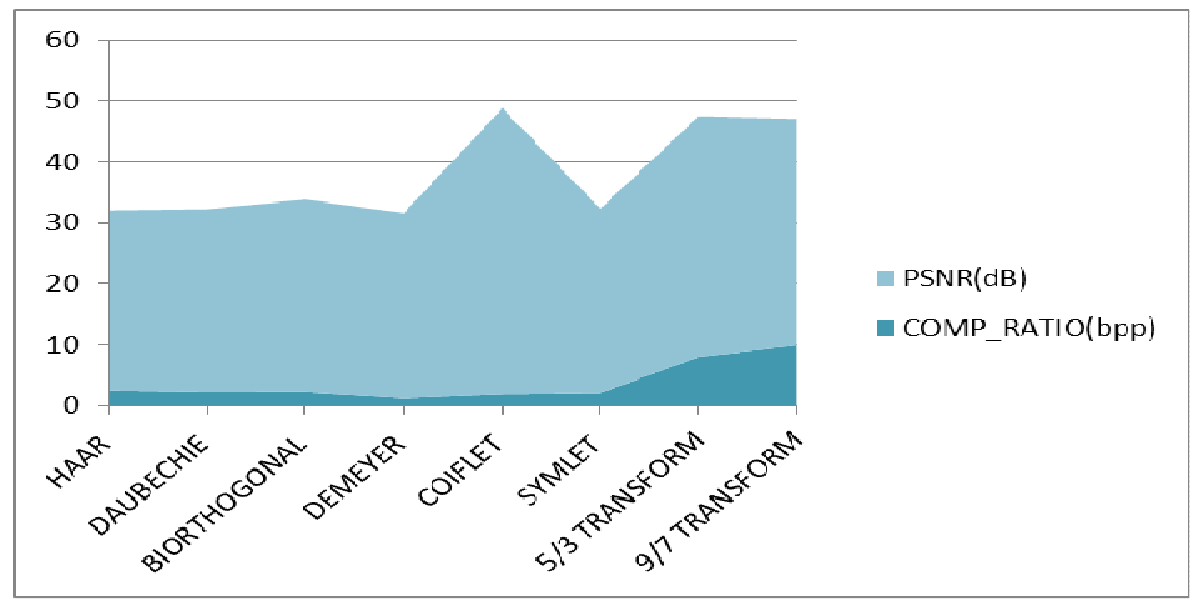

FIGURE 2: Performance of different Wavelets on “Cameraman” image. 
Signal \& Image Processing : An International Journal (SIPIJ) Vol.3, No.2, April 2012

\section{CONClusions \& Future SCOPE}

In this paper, Hand designed and lifting based wavelet transforms are compared. Haar wavelet, Daubechie wavelet, Demeyer wavelet, Coiflet wavelet and Symlet wavelets are considered under Hand designed wavelet category; 5/3 and 9/7 lifting based wavelets are considered under Lifting based wavelet transform category. Except Coiflet wavelet, all the Hand designed wavelets produced less PSNR around $30 \mathrm{~dB}$ and less compression ratio around $2 \mathrm{bpp}$.

Coiflet wavelet produced high PSNR around $47 \mathrm{~dB}$, but at low compression ratio in the ranges of only $2 \mathrm{bpp}$. The lifting based wavelet transforms, on the other hand, produced high PSNR and high compression ratio. The PSNR is almost above $40 \mathrm{~dB}$ and compression ratio is above $7 \mathrm{bpp}$. From the above discussion, it is evident that the lifting based wavelets outperform the traditional or hand-designed wavelets.

The future scope of the presented work can be summarized as follows.

- The techniques can be used along with modified SPIHT for improved image compression.

- The techniques can be extended for video compression.

- The techniques can be extended for any other image processing applications for better results.

\section{REFERENCES}

[1] I. JTC1/SC29/WG1, "JPEG 2000 - lossless and lossy compression of continuous- tone And bi-level still images", Part 1: Minimum decoder. Final committee draft, Version1.0., March 2000.

[2] G.Wallace, “The JPEG still picture compression standard”, IEEE TCE, 38, 1992.

[3] A Selective data pruning-based compression using high-order edge-directed interpolation Dung T., Troung Q.Nguyen., IEEE Transaction on Image Processing fed 2010.

[4] Independent JPEG Group, version 6a:http://www.ijg.org.

[5] G. K. Wallace, "The JPEG still picture compression standard”, in IEEE Transactions on Circuits and Systems for Video Technology, vol. 6, June 1996.

[6] O. K. Al-Shaykh, "JPEG-2000: A new still image compression standard", in Conference Record of Thirty-Second Asilomar Conference on Signals Systems and Computers, Vol.1, pp. 99-103, 1998.

[7] JPEG2000, http://www.jpeg.org/JPEG2000.htm.

[8] Moving Picture Expert Group Standard, http://www.mpeg. org/MPEG/index.html.

[9] L.Szu Y.Sheng, "Wavelet processing and optics”, IEEE 84(5), 1996, pp. 720-732 Imaging, October 1999, Vol.7 (4).

[10] M.Borland, "Wavelet in image communication”, Elsevier 1994.

[11] Charles Hsu et al., 1999, "Wavelet brass boards for live video via ratio", Journal of Electronic Imaging, Vol. 7(4).

[12] O.Strome et al, 1997, "Study of wavelet decompositions for image/video compression by software codecs", IEEE, pp. 125-132. 
Signal \& Image Processing : An International Journal (SIPIJ) Vol.3, No.2, April 2012

[13] W. Sweldens, 1997, "The lifting scheme: A construction of second generation wavelets", SIAM J. Math. Anal., Vol.29 (2), pp.511-546.

[14] I. Daubechies, W. Sweldens, 1998, "Factoring wavelet into lifting steps", J. Fourier Anal. Appl., Vol.4 (3), pp.247-269.

[15] D. Taubman “Adaptive non-separable lifting transforms for image compression”, in Proc. Int. Conf. Image Processing, Kobe, Japan, Oct.1999.

[16] A. Gouze, M. Antonini, M. Barland, 2000, "Quincunx lifting scheme for lossy image compression", IEEE, pp. 665-668.

[17] A. Gouze, M. Antonini, M. Barland, Benoit Macq, 2004, "Design of signal -adapted multidimensional lifting scheme for lossy coding”, IEEE Trans. on Image Processing, Vol.13 (12), pp. 1589-1603.

[18] R.L. Caypoole, G.M. Davis, W. Sweldens, and R.Gboranuk, 2003, "Nonlinear wavelet transforms for image coding via lifting”, IEEE Trans. on Image Processing, Vol.12,pp. 1149-1459.

[19] Ömer Nezih Gerek, A.Enisçetin, 2000, “Adaptive polyphase decomposition structure for image compression”, IEEE Trans. on Image Processing, Vol.9 (10).

[20] N.V. Boulgouris, Dimitrios Tzavaras and Michael Gerassiomos Strintzis, 2001, "Lossless image compression based on optimal prediction, adaptive lifting and Conditional arithmetic coding", IEEE Transaction on Image Processing, Vol.10 (1), pp. 1-14.

[21] G. Piella, H.J.A.M. Heijmans “Adaptive lifting scheme with perfect reconstruction”, IEEE Trans. on Signal Processing, Vol.50 (7), July 2002, pp. 1620-1630.

[22] G. Piella, B. Pesquet-Popescu, H.J.A.M. Heijmans, 2002, “Adaptive update lifting with a decision rule based on derivative filters", IEEE Signal Processing Letters, Vol.9 (10).

[23] K. A. Kotteri, A. E. Bell, and J. E. Carletta, "Design of multiplierless, high-performance, wavelet filter banks with image compression applications," IEEE Tran. On Circuits and Systems-I, vol. 51, no. 3, pp. 483.494, Mar. 2004

\section{AUTHORS}

Dr. B. Eswara Reddy Graduated in B.Tech. (CSE) from Sri Krishna Devaraya University in 1995. He received Masters Degree in M.Tech.(Software Engineering), from JNT University, Hyderabad, in 1999. He received Ph.D in Computer Science \& Engineering from JNT University, Hyderabad, in 2008. He served as Assistant Professor from 1996 to 2006. He is working as Associate Professor in CSE Dept., since 2006 and currently acting as Head of CSE Dept. at JNTUACE, Anantapur. He has more than 30 Publications in various International Journals and Conferences. He is one of the author's

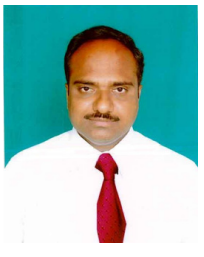
of the text book titled Programming with Java published by Pearson/Sanguine Publishers. His research interests include Pattern Recognition \& Image Analysis, Data Warehousing \& Mining and Software Engineering. He is a life member of ISTE, IE, ISCA and member of CSI and IEEE

K.Venkata Narayana received his Master of Computer Applications (MCA) Degree from JNTU, Hyderabad in 2007. He received M.Phil Degree from Sri KrishnaDevaraya University in 2009 At Present he is working as a Programmer in SKUniversity College of Engg \& Tech,SKUniversity Anantapur.And He is Pursuing his M.Tech(CSE) from JNTUACEA,Anantapur.His research interest is Image Processing, Image Compression and Data Mining.

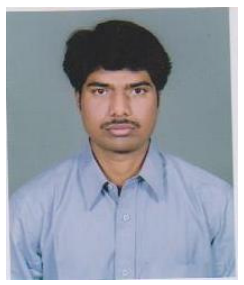

